\title{
Phenotypic distribution of the stem-like melanoma initiating state depends on CDKN2A status
}

Yuntian Zhang ${ }^{1}$, Maike M. K. Hansen ${ }^{2}$, Miroslav Hejna ${ }^{3}$, Leor S. Weinberger ${ }^{4,5}$, and Robert L. Judson-Torres ${ }^{1,6,7, *}$

${ }^{1}$ Helen Diller Family Comprehensive Cancer Center, University of California, San Francisco, CA 94158, USA

${ }^{2}$ Radboud University, Institute for Molecules and Materials, Heyendaalseweg 135, 6525 AJ Nijmegen, the Netherlands

${ }^{3}$ Department of Physics and Carl R. Woese Institute for Genomic Biology, University of Illinois at Urbana-Champaign, Urbana, IL 61801, USA

${ }^{4}$ Gladstone Center for Cell Circuitry, Gladstone Institutes, San Francisco, CA 94158, USA

${ }^{5}$ Departments of Pharmaceutical Chemistry and Biochemistry and Biophysics, University of

California, San Francisco, CA 94158, USA

${ }^{6}$ Huntsman Cancer Institute, University of Utah, Salt Lake City, UT 84112, USA

${ }^{7}$ Department of Dermatology, University of Utah School of Medicine, Salt Lake City, UT 84103, USA

* Correspondence and Lead Contact: judsontorreslab@gmail.com

The authors declare no conflicts of interest. 


\begin{abstract}
Many cancers contain distinct tumor-initiating cell populations. However, the existence of distinct stem-like melanoma initiating cells and their contribution to tumorigenesis remains contested $^{1-5}$. To identify this cell population in melanoma, we used quantitative single cell approaches linking gene expression, genotype and phenotype in melanoma cells, and observed that bidirectional interconversion between tumor-initiating and differentiated non-tumorigenic states establishes distinct phenotypic equilibria dependent on genotype. Genetic loss of the CDKN2A locus corresponds to a uniform adoption of a neural crest stem cell (NCSC) like tumorinitiating state. Exposure to a putative chemopreventative $\alpha$-melanocyte stimulating hormone ( $\alpha \mathrm{MSH}$ ) analog can substitute for CDKN2A loss and shift phenotype distribution toward the tumor-initiating state. Alarmingly, in vivo application of the analog is sufficient to induce tumorigenesis in otherwise non-tumorigenic populations. Our results demonstrate that dynamic stemness in melanoma is dependent on secondary mutation status, highlighting the need to incorporate genomic characterization when developing potential chemopreventative agents.
\end{abstract}

\title{
Main
}

Melanoma cells exhibit substantial phenotypic plasticity ${ }^{6}$. A variety of putative mutually exclusive transcriptional programs orchestrate this plasticity. The poles of one reported axis comprise a proliferative program driven by MITF and an invasive program driven by POU3F2 
and $\mathrm{AXL}^{6-9}$. Separate studies have also identified cancer stem cell (CSC) or tumor initiating cell (TIC) populations marked by $\mathrm{AXL}^{10}, \mathrm{ABCB}^{1}, \mathrm{KDM} 5 \mathrm{~B}(\mathrm{JARID1B})^{11}, \mathrm{NGFR}(\mathrm{CD} 271)^{2,12}, \mathrm{PROM} 1$ $(\mathrm{CD} 133)^{13}, \mathrm{JUN}^{14}$ and SOX10 ${ }^{12}$, among others. Differential adoption of the stem cell program, proliferative program, and the invasive program influences critical processes at each step of melanoma progression. Consequently, controlled pharmacological manipulation of phenotypic states is a tempting strategy for preventing melanoma initiation, progression and recurrence ${ }^{6}$.

The development of agents that reproducibly influence phenotypic state for clinical administration has been hindered by an incomplete understanding of the complex interactions between genome, microenvironment, transcriptional state and phenotype. For example, studies in human cells have been unable to consistently reproduce the inhibitory relationship between MITF and POU3F2 observed in mouse cells ${ }^{7,15-22}$ and only a subset of lines demonstrate anti-correlative expression ${ }^{22,23}$. Further, markers of TICs and CSCs are not reproducible across studies, are demonstrably unstable, or are non-exclusive $e^{3-5,11,24}$. Understanding the fundamental variables that underlie these discrepancies is required for therapeutic strategies that seek to exploit phenotype manipulation. The mutational burden of melanoma is among the highest of all human cancers ${ }^{25}$. It is thus reasonable to hypothesize that the observed heterogeneity in gene expression : phenotype relationships is due to differences in genetic landscape. Although previous studies have found no correlations between driver mutation status and the above discrepancies, the influence of secondary mutations remains uncharacterized. We thereafter sought to investigate coupled gene expression and phenotype distributions within a panel of cell lines with established genotypes. 
Given its role in the proliferation-invasion axis, we investigated the uniformity and dynamics of POU3F2 expression within clonal cell populations. As the CDKN2A locus is both the most frequent site for secondary mutations in melanoma ${ }^{26}$ and regulates POU3F2 expression ${ }^{21}$, we assessed POU3F2 expression heterogeneity in three CDKN2A retaining and three CDKN2A null cell lines ${ }^{21,27-30}$ using quantitative immunofluorescent (IF) (Fig. S1a-b) and single molecule fluorescence in situ hybridization (smFISH) imaging. Expression in CDKN2A retaining lines exhibited a broad distribution whereas in CDKN2A null lines the distribution was uniformly low (Fig. 1a-b, Fig. S1c-d). To determine whether POU3F2 expression heterogeneity was genetic or phenotypic, we expanded clonal populations of CDKN2A retaining cells and monitored POU3F2 expression over time. Regardless of the initial profile, the broad distribution characteristic of the parental populations was re-established over 2-3 months in culture (Fig. S1e-f), indicating a phenotypic equilibrium is selected during the culture of clonal populations.

We engineered a mCherry-based reporter of POU3F2 promoter activation. Clonally expanded reporter cells presented heterogeneous mCherry expression associated with distinct morphologies (Fig. 1c, Fig. S2a-b). FACS-isolated reporter low cells expressed 2-3 fold less POU3F2 protein (Fig. S2C-d) and mRNA (Fig. 1d, Fig. S2e-g). smFISH confirmed a significant correlation between POU3F2 and mCherry mRNA levels (Fig. S2g). The distribution of POU3F2 mRNA count in the parental lines presented as a combination of those of the isolated reporter low and reporter high populations (Fig. 1d). To determine whether the two distinct POU3F2 transcriptional states are interconvertible, single cells were isolated and clonally expanded. Live 
quantitative imaging did not capture interconversions within single cell cycles (Fig. S3a).

Cultures likewise were predominantly uniform after short-term expansion (15-20 divisions) (Fig. 1e-i,iii and Fig. S3b) but subpopulations emerged with extended expansion (>100 divisions) (Fig. 1e-ii,iv). In CDKN2A retaining lines interconversion was both bidirectional and serial: uniform reporter high populations begot low populations and vice versa (Fig. 1e and Fig. S3b). In contrast, CDKN2A null clones remained uniform throughout extended culture (Fig. S3c). We conclude the two transcriptional states interconvert bidirectionally in CDKN2A retaining lines and are inheritable over several cell divisions - dynamics that are consistent with an intermediate memory timescale ${ }^{31}$.

To characterize the two states, we FACS-isolated and profiled transcriptomes of each population. Unsupervised hierarchical clustering classified each state based upon 3014 genes that were differentially expressed between the two groups (adj. p-value <0.005) (Fig. 2a, Fig. S3d and Table S1). The reporter low cells were characterized by expression of AXL, JUNB, ACP1, PTEN and MYC (Fig. 2a) and enrichment for $\operatorname{NCSC}^{32,33}$, invasive ${ }^{10,34}$, and mesenchymal programs $^{35}$ and MYC, JUN, and SMAD4 targets $^{36}$ (Fig. 2b and Table S2). In contrast, other previously reported TIC or CSC markers were either unchanged or displayed greater expression in the reporter high population (Fig. 2a-ii). Reporter low cells were depleted of the MITF, proliferation and melanocyte differentiation programs (Fig. 2a-b), as well as genes frequently genetically disrupted at the onset of melanoma invasion, such as CDKN2A, ARID1A and ARID1B $^{37}$ (Fig. 2a-iii). MITF protein expression varied dramatically in the baseline expression across lines, but the reporter low population consistently expressed less MITF and was less 
pigmented (Fig. 2c-d). Interestingly, both $\mathrm{BRAF}^{\mathrm{V} 600 \mathrm{E}}$ and the CDKN2A product INK4 displayed a similar pattern of heterogenous baseline expression but consistent downregulation in each reporter low population (Fig. 2c).

Using quantitative phase cytometry, we conducted single cell phenotype profiling ${ }^{38,39}$ (Fig. 2e), and unexpectedly identified the POU3F2 reporter high population to be moderately less motile than the reporter low counterparts (Fig. 2f) - a result confirmed via scratch assay (Fig. 2g). To determine whether either population were enriched for TICS, FACS-isolated reporter low and high cells were implanted subcutaneously into NOD scid gamma mice and tumor growth was monitored for five weeks (Fig. 2h). Mice implanted with reporter low cells formed tumors in $95.5 \%$ of cases, as compared to $14.3 \%$ of those implanted with reporter high cells (Fig. $2 \mathrm{i}$ ). Supplementing in just 1-4\% of reporter low cells into a predominantly reporter high population significantly increased tumorigenicity by four folds. We conclude the transcriptional program characterized by low POU3F2, MITF, INK4A, and BRAF, but high AXL, MYC, JUNB, and PTEN marks a plastic tumor initiating state (TS) that is distinct from both poles of the previously reported proliferation-invasion axis as well as ABCB5, JARID1B, CD271, or CD133 positive CSCs.

Genetic disruption of CDKN2A induces melanoma initiation in RAS or RAF activated melanocytes ${ }^{21,40-43}$. Since CDKN2A-null melanoma lines presented a uniform distribution of reporter low cells (Fig. 1a, Fig. S1d) and CDKN2A-retaining reporter low cells expressed less CDKN2A product then reporter high counterparts (Fig. 2a,c), we reasoned that loss of this tumor suppressor shifts the equilibrium toward the TS. We employed our previously 
characterized CRISPR/CAS9 engineering platform for deletion of CDKN2A exon 2 and observed a significant and rapid shift in phenotypic equilibrium, with a $20 \%$ increase in the TS population (Fig. 3a). Since $40 \%$ of melanomas retain and express at least one CDKN2A locus ${ }^{21}$, we next investigated whether other factors such as extrinsic stimuli could either mimic this shift, potentially inducing tumorigenesis, or elicit an opposing shift, potentially preventing tumorigenesis. Given the development of $\alpha \mathrm{MSH}$ analogs as chemopreventative agents ${ }^{44,45}$, we first asked whether exposure to $\alpha \mathrm{MSH}$ or analogs would disrupt the phenotypic equilibrium. Reporter high cells were treated with $1 \mu \mathrm{M} \alpha \mathrm{MSH}, \beta \mathrm{MSH}, \gamma \mathrm{MSH}$, or synthetic MSH analogs Melanotan 1 (MT1) and Melanotan2 (MT2) - and analyzed via flow cytometry after 1h (Fig. 3b). MT2 induced a surprising induction of more motile reporter low cells (Fig. 3b-c, Fig. S4a,b). TS induction was specific to MT2, despite a comparable increase in phospho-CREB from $\alpha M S H$, MT1, and MT2 exposure (Fig. 3d). The TS induction was consistent, but the magnitude of the shift was minor and variable - an approximate $0.05-1 \%$ increase in TS. We investigated whether this nuanced shift in phenotypic equilibrium conveyed a meaningful change in tumorigenicity. TS-depleted and TS-purified subpopulations constitutively expressing luciferase were implanted subcutaneously into mice which then received intraperitoneal injections of MT2 $(1 \mathrm{mg} / \mathrm{kg})$ or saline twice a week starting the day of implantation (Fig. 4a). The luminescence of each implantation site was monitored over five weeks, followed by imaging the brain, lungs, and liver (Fig. 4b). Treatment with MT2 significantly increased both tumorigenicity and metastatic burden of mice implanted with otherwise non-tumorigenic populations (Fig. 4c-d). 
In summary, we demonstrate a melanoma stem-like TS arises from differentiated cells so that clonal subpopulations return toward phenotypic equilibria over extended time. We support a hybrid model of progression in which clonal evolution orchestrates 'dynamic stemness' ${ }^{6}$, offering an explanation for the conflicting reports regarding TIC stability. We observed that the coincident absence of both POU3F2 and MITF expression mark an NCSC-like TS, mirroring early melanocyte development (Fig. S5a-b) ${ }^{46,47}$. We propose that each individual cell within a melanoma population can occupy a myriad of semi-stable transcriptional states and the percentage of cells occupying the TS is influenced by both secondary mutations (e.g. CDKN2A status) and chemical perturbation (e.g. MT2). We therefore speculate that for chemopreventative candidates to exhibit clinical utility, they must invariably induce an exclusively non-tumorigenic distribution regardless of mutational status.

The potential of $\alpha \mathrm{MSH}$ analogs as chemopreventative melanoma agents has been established $^{44}$. Our study demonstrates that exposure to MT2, itself an analog of $\alpha M S H$, is also tumorigenic dependent on the initial phenotypic distribution and genotype of the tumor cell population, consistent with anecdotal clinical case reports that associate injection of MT2, but rarely other MC1R agonists, with nevus growth and melanoma initiation ${ }^{48}$. As synthetic analogs of $\alpha \mathrm{MSH}$ are further developed, it will be important to characterize their effect on the distribution of tumorigenic subpopulations on different genetic backgrounds.

In conclusion, we demonstrate that cancer cell phenotypic equilibria depend on the mutational landscape. Subtle shifts in phenotypic equilibria may promote tumor progression through 
induction of minor stem-like subpopulations, highlighting the importance of quantitative approaches with single-cell resolution. We also suggest a broader role for the tumor suppressor, $C D K N 2 A$, in regulating global cell state plasticity in addition to its established role as a cell cycle inhibitor. This study improves our understanding of how secondary mutation status alters cell state plasticity and, consequently, the effect of environmental cues on tumor progression.

\section{Acknowledgments:}

We thank Rachel Belote, Hanlin Zeng, and Rajan Kulkarni for critical reading of this manuscript. This manuscript has been edited by Life Science Editors. We acknowledge the use of the $\mathrm{HCl}$ Shared Resources for Research Informatics, High-Throughput Genomics and Bioinformatics Analysis, and the Flow Cytometry supported by P30CA042014 awarded to $\mathrm{HCl}$ from the National Cancer Institute. This work was supported by NIH award DP5 OD019787 (to RL), the Program for Breakthrough Biomedical Research Sandler Fellowship (to RL), and Elsa U. Pardee Foundation research award CA-0122861 (to YZ) for financial support.

\section{Author Contributions:}

Yuntian Zhang: Conceptualization, Investigation, Formal analysis, Writing - original draft, Visualization, Funding acquisition; Maike M. K. Hansen: Investigation, Formal analysis, Writing review \& editing, Visualization; Miroslav Hejna: Formal analysis; Leor S. Weinberger: Conceptualization, Supervision, Writing - review \& editing; Robert L. Judson-Torres: 
Conceptualization, Investigation, Formal analysis, Writing - original draft; Visualization, Supervision, Funding acquisition.

\section{Figure Legends}

Figure 1: Melanoma cells undergo bidirectional interconversion between POU3F2 expression states

a) Images (top) and quantification (bottom, arbitrary fluorescence units of individual cells) of POU3F2 IF staining of CDKN2A retaining ( $\mathrm{n}=898$ cells) or CDKN2A null ( $\mathrm{n}=1474$ cells) lines (more lines shown in Fig. S1c,d). Red arrowheads indicate cells with poor POU3F2 expression in $C D K N 2 A$ retaining line. Exposure time increased in $C D K N 2 A$ null line representative image to visualize uniformity. Scale bar $=20 \mu \mathrm{m}$. b) Images (top) and mRNA count of individual cells (bottom) of single molecule FISH probed for POU3F2 mRNA in CDKN2A retaining $(\mathrm{n}=3$ lines, 1195 cells) or CDKN2A null ( $\mathrm{n}=3$ lines, 1440 cells) lines. Scale bar $=10 \mu \mathrm{m} \mathrm{c}$ ) Illustration of the reporter construct expressing mCherry with a nuclear localization signal (NLS) under the control of the POU3F2 promoter (top) and a representative image of clonal 501Mel cells expressing the reporter (bottom). Scale bar $=50 \mu \mathrm{m}$. d) Quantification of POU3F2 transcripts in parental and isolated reporter high and reporter low 501MEL cells. e) Schematic (top) and representative FACS plots (bottom) of experimentally tracking bidirectional interconversion of 501MEL reporter cells. Roman numerals indicate corresponding experimental time points and plots (more lines shown in Fig. S3). P values generated from unpaired T tests. 
Figure 2: Low POU3F2 marks neural crest stem-like tumor initiating cells

a) Unsupervised hierarchical clustering of isolated reporter high and reporter low 501MEL cells based upon all expressed genes. Shown are heat-maps of select genes involved in (i) phenotype switching; (ii) melanoma stem cell identification; and (iii) melanoma progression. Adjusted P value for differential expression indicated by italics $(>0.05)$, bold $(<0.05)$, and underline $(<0.005)$. Full differentially expressed gene list is in Supplementary Table 1. b) Visualization of gene set enrichment analyses of differential expression analysis. X-axis reports ranked gene expression and dots represent gene associated with indicated gene set. Normalized enrichment score and nominal $p$ value are shown at the right. $*=p<0.05 ; * *=p<0.005 ; * * * *=p<0.00005 . c)$ Western blot analysis of isolated reporter high $(\mathrm{H})$ and reporter low $(\mathrm{L})$ cells from indicated cell lines using indicated antibodies. d) Images of pigmentation from pelleted isolated reporter high and reporter low cells. e) Frames from time-lapse QPI of reporter cells with mCherry expression. Scale bar $=50 \mu \mathrm{m} . \mathrm{f}$ ) Cell motility over $24 \mathrm{~h}$. Violin plots represent distribution of indicated cell numbers from three independent experiments compared with two-tailed unpaired t-Test. g) Scratch assay comparing migration of reporter high and reporter low cells. Graph plots gap width over time as a percent of width at time point $0 \mathrm{hr}$. Error bars indicate standard deviation over three independent experiments. $h$,i) Schematic ( $h$ ) and results (i) of in vivo implantation study. FACS was used to generate populations of 501MEL cells with indicated percentages of reporter low cells, which were subsequently implanted into immunecompromised mice. The percentage of mice that were successfully engrafted is presented. Data are compared by Chi-square and Fisher's exact t-Test. 
Figure 3: CDKN2A loss or Melanotan 2 exposure induce the tumor initiating state

a) Schematic (top) of CDKN2A knockout (KO) experimental strategy and change in reporter low population (bottom). b) Schematic (top) of experimental strategy and change (bottom) in

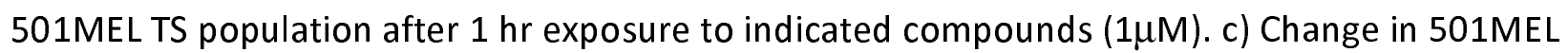
TS population after $1 \mathrm{hr}$ exposure with DMSO (vehicle) or melanotan 2 (more lines shown in Fig. S4). d) Representative western blots of 501MEL cells using indicated antibodies after treatments from (b). MT=melanotan. Error bars indicate standard deviation of mean $(\mathrm{n}=3)$. $\mathrm{P}$ values generated from unpaired $\mathrm{T}$ tests

Figure 4: Melanotan 2 induces melanoma tumorigenesis in vivo a) Schematic of experimental design. TS (reporter low) and TS-depleted (reporter high) populations of 501MEL reporter cells were FACS isolated and implanted into immunecompromised mice. Mice injected with Melanotan $2(1 \mathrm{mg} / \mathrm{kg})$ or saline twice a week for five weeks starting on day of implantation were subjected to bioluminescent live imaging as indicated. b) Representative bioluminescent imaging of mice at indicated time points and harvested organs at the end of week five. c) Take rate after implantation. Data are presented as percentage compared by Chi-square and Fisher's exact t-Test. d) Total metastatic burden presented as arbitrary units from total organ luciferase activity. Data are presented as minimum to maximum box and whiskers plot compared by unpaired Mann Whitney test.

Figure S1. POU3F2 exhibits heterogenous expression in CDKN2A-retaining melanoma lines. Related to Figure 1. 
a-b) Images (a) and quantification (b, arbitrary fluorescence units of individual cells) of POU3F2 IF staining with shRNA mediated POU3F2 knockdown (shPOU3F2), no treatment (parental), POU3F2 overexpression (OE POU3F2) and no primary antibody (secondary), demonstrating quantitative IF. Scale bar $=20 \mu \mathrm{m} . \mathrm{c}-\mathrm{d})$ Images (c) and quantification (d, arbitrary fluorescence units of individual cells) of POU3F2 IF staining of indicated cell lines as in Fig. 1a. Red arrowheads indicate cells with poor POU3F2 expression in CDKN2A retaining lines. Exposure time increased in CDKN2A null line representative images for visibility. Scale bar $=20 \mu \mathrm{m} . \mathrm{P}$ value calculated with unpaired T test. e-f) Images of POU3F2 IF in 501MEL single cell clonal expansions at 1 month, 2 months and 3 months in culture (e) and quantification of the intensity of POU3F2 IF ( $f$, arbitrary fluorescence units of individual cells) from independent clones measured after 2 weeks (before arrow) then 2 months (after arrow) expansion. Dotted line is for reference.

Figure S2. POU3F2 low cells are a morphologically distinct population. Related to Figure 1c. a) Imaging of reporter high and reporter low cells using fluorescent labels (DAPI and CellBrite green, left) or DHC (right). Scale bar $=50 \mu \mathrm{m}$. b) The distribution of cells (top) and accuracy of classification (bottom) using a linear discriminant analysis trained with DHC morphologic features. c-d) Image (c) and quantification (d) of western blotting for POU3F2 using isolated reporter high (RH, Red) and reporter low (RL, grey) cells from WM793, 501MEL, and SK-MEL-28. e) Real-time PCR quantification of POU3F2 mRNA from isolated reporter high (red) and reporter low (grey) cells. f) Images of 501Mel cells probed with single molecule FISH for POU3F2 and mCherry transcripts. Scale bar $=10 \mu \mathrm{m} . \mathrm{g}$ ) The number of mCherry transcripts plotted against 
the number of POU3F2 transcripts in each cell of smFISH analysis. Two-tailed P value from Pearson correlation $(g)$ or unpaired $T$ test $(b, d, e)$.

Figure S3. Bidirectional interconversion of POU3F2 states occurs at intermediate time-scale. Related to Figure 1 and 2.

a) Time-lapse DHC imaging of 501MEL reporter low (top row) and reporter high (bottom row) cells before, after, and in between consecutive M-phases. States can be identified with $96.05 \%$ accuracy (Fig. S2b). State switching was not observed during a standard cell cycle. Scale bar = $50 \mu \mathrm{m}$. Numbers indicate hours from first image. b-c) Experimental tracking of bidirectional interconversion between reporter low and reporter high states, as in Fig. 1e, for indicated $C D K N 2 A$ retaining (b) and $C D K N 2 A$ null (c) cell lines. d) Volcano plot showing genes differentially expressed in reporter high and reporter low cells. Full differentially expressed gene list in Supplementary Table 1.

Figure S4. Assays for cell behavior when considering total- or sub-populations. Related to Figure 3.

a) Change in 501MEL reporter low population after $1 \mathrm{hr}$ exposure to melanotan $2(1 \mu \mathrm{M})$. b) Schematic (top) of experimental design and results (bottom) of scratch assay to assess the effect of melanotan 2 (MT2) treatment on predominantly reporter high 501Mel cells when considering the full population or just the reporter low subpopulation. The total population (blue) then the isolated MT2-induced reporter low population (grey) were assayed. When measured as a population average, the effect of MT2 exposure on the predominately reporter 
high population was to decrease motility (blue), consistent with previous reports. Treatment also induced a small population of reporter low cells which, when assayed as an independent subpopulation, are more motile (grey).

Figure S5: Proposed model of bidirectional interconversion between multiple transcriptional states.

a) Schematic of POU3F2 expression levels during melanocyte development. Represents compilation of previous reports and data presented here (green box). POU3F2 is most highly expressed in migratory melanoblasts and is poorly expressed in both neural crest stem cells and differentiated melanocytes. b) The relationship among the expression level of indicated genes and different melanoma cell states. The NCSC-like tumor initiating state is characterized by low BRAF $^{\mathrm{V} 600 \mathrm{E}}$, POU3F2, MITF, and INK4A and high AXL expressions. The previously reported MITFPOU3F2 proliferation-invasion axis is established in more differentiated cells. When CDKN2A is retained, transcriptional states exhibit intermediate stability. The absence of $C D K N 2 A$ permits a greater degree of plasticity, resulting in a uniform population in selective conditions.

\section{References}

1. Schatton, T. et al. Identification of cells initiating human melanomas. Nature 451, 345349 (2008).

2. Boiko, A. D. et al. Human melanoma-initiating cells express neural crest nerve growth factor receptor CD271. Nature 466, 133-137 (2010).

3. Quintana, E. et al. Efficient tumour formation by single human melanoma cells. Nature $456,593-8$ (2008). 
4. Quintana, E. et al. Phenotypic Heterogeneity among Tumorigenic Melanoma Cells from Patients that Is Reversible and Not Hierarchically Organized. Cancer Cell 18, 510-523 (2010).

5. Boyle, S. E. et al. CD271 Expression on Patient Melanoma Cells Is Unstable and Unlinked to Tumorigenicity. Cancer Res. 76, 3965-3977 (2016).

6. Arozarena, I. \& Wellbrock, C. Phenotype plasticity as enabler of melanoma progression and therapy resistance. Nat. Rev. Cancer 19, 377-391 (2019).

7. Hoek, K. S. et al. In vivo switching of human melanoma cells between proliferative and invasive states. Cancer Res. 68, 650-656 (2008).

8. Fane, M. E., Chhabra, Y., Smith, A. G. \& Sturm, R. A. BRN2, a POUerful driver of melanoma phenotype switching and metastasis. Pigment Cell and Melanoma Research 32, 9-24 (2019).

9. Strub, T. et al. Essential role of microphthalmia transcription factor for DNA replication, mitosis and genomic stability in melanoma. Oncogene 30, 2319-2332 (2011).

10. Widmer, D. S. et al. Systematic classification of melanoma cells by phenotype-specific gene expression mapping. Pigment Cell Melanoma Res. 25, 343-353 (2012).

11. Roesch, A. et al. A Temporarily Distinct Subpopulation of Slow-Cycling Melanoma Cells Is Required for Continuous Tumor Growth. Cell 141, 583-594 (2010).

12. Civenni, G. et al. Human CD271-positive melanoma stem cells associated with metastasis establish tumor heterogeneity and long-term growth. Cancer Res. 71, 3098-3109 (2011).

13. Monzani, E. et al. Melanoma contains CD133 and ABCG2 positive cells with enhanced tumourigenic potential. Eur. J. Cancer 43, 935-946 (2007). 
14. Ramsdale, R. et al. The transcription cofactor c-JUN mediates phenotype switching and BRAF inhibitor resistance in melanoma. Sci. Signal. 8, ra82-ra82 (2015).

15. Thomson, J. et al. The brn-2 gene regulates the melanocytic phenotype and tumorigenic potential of human melanoma cells. Oncogene 11, 691-700 (1995).

16. Fane, M. E. et al. NFIB Mediates BRN2 Driven Melanoma Cell Migration and Invasion Through Regulation of EZH2 and MITF. EBioMedicine 16, 63-75 (2017).

17. Simmons, J. L., Pierce, C. J., Al-Ejeh, F. \& Boyle, G. M. MITF and BRN2 contribute to metastatic growth after dissemination of melanoma. Sci. Rep. 7, 10909 (2017).

18. Goodall, J. et al. Brn-2 represses microphthalmia-associated transcription factor expression and marks a distinct subpopulation of microphthalmia-associated transcription factor-negative melanoma cells. Cancer Res. 68, 7788-94 (2008).

19. Boyle, G. M. et al. Melanoma cell invasiveness is regulated by miR-211 suppression of the BRN2 transcription factor. Pigment Cell Melanoma Res. 24, 525-537 (2011).

20. Thurber, A. E. et al. Inverse expression states of the BRN2 and MITF transcription factors in melanoma spheres and tumour xenografts regulate the NOTCH pathway. Oncogene 30, 3036-3048 (2011).

21. Zeng, H. et al. Bi-allelic Loss of CDKN2A Initiates Melanoma Invasion via BRN2 Activation. Cancer Cell 34, 56-68.e9 (2018).

22. Pierce, C. J. et al. BRN2 expression increases anoikis resistance in melanoma. Oncogenesis 9, (2020).

23. Smith, M. P. et al. A PAX3/BRN2 rheostat controls the dynamics of BRAF mediated MITF regulation in MITFhigh/AXLlow melanoma. Pigment Cell Melanoma Res. 32, 280-291 
(2019).

24. Madjd, Z. et al. Expression of CD133 cancer stem cell marker in melanoma: A systematic review and meta-analysis. International Journal of Biological Markers 31, e118-e125 (2016).

25. Bastian, B. C. The Molecular Pathology of Melanoma: An Integrated Taxonomy of Melanocytic Neoplasia. Annu. Rev. Pathol. Mech. Dis. 9, 239-71 (2014).

26. Shain, A. H. et al. The genetic evolution of melanoma from precursor lesions. N. Engl. J. Med. (2015). doi:10.1056/NEJMoa1502583

27. Giard, D. J. et al. In vitro cultivation of human tumors: Establishment of cell lines derived from a series of solid tumors. J. Natl. Cancer Inst. 51, 1417-1423 (1973).

28. Stark, M. \& Hayward, N. Genome-wide loss of heterozygosity and copy number analysis in melanoma using high-density single-nucleotide polymorphism arrays. Cancer Res. 67, 2632-2642 (2007).

29. Castellano, M. et al. CDKN2A/p16 is inactivated in most melanoma cell lines. Cancer Res. 57, 4868-75 (1997).

30. Poliseno, L. et al. Deletion of PTENP1 pseudogene in human melanoma. Journal of Investigative Dermatology 131, 2497-2500 (2011).

31. Shaffer, S. M. et al. Memory sequencing reveals heritable single cell gene expression programs associated with distinct cellular behaviors. bioRxiv 379016 (2018). doi:10.1101/379016

32. Tirosh, I. et al. Dissecting the multicellular ecosystem of metastatic melanoma by singlecell RNA-seq. Science (80-. ). 352, 189-196 (2016). 
33. Tsoi, J. et al. Multi-stage Differentiation Defines Melanoma Subtypes with Differential Vulnerability to Drug-Induced Iron-Dependent Oxidative Stress. Cancer Cell 33, (2018).

34. Hoek, K. S. et al. Novel MITF targets identified using a two-step DNA microarray strategy. Pigment Cell Melanoma Res. 21, 665-676 (2008).

35. Lamouille, S., Xu, J. \& Derynck, R. Molecular mechanisms of epithelial-mesenchymal transition. Nature Reviews Molecular Cell Biology 15, 178-196 (2014).

36. Lachmann, A. et al. ChEA: transcription factor regulation inferred from integrating genome-wide ChIP-X experiments. Bioinformatics 26, 2438-44 (2010).

37. Shain, A. H. et al. Genomic and Transcriptomic Analysis Reveals Incremental Disruption of Key Signaling Pathways during Melanoma Evolution. Cancer Cell 34, 45-55.e4 (2018).

38. Hejna, M., Jorapur, A., Song, J. S. \& Judson, R. L. High accuracy label-free classification of kinetic cell states from holographic cytometry. bioRxiv 1, 48-51 (2017).

39. Zhang, Y. \& Judson, R. L. Evaluation of holographic imaging cytometer holomonitor M4 ${ }^{\circledR}$ motility applications. Cytom. Part A (2018). doi:10.1002/cyto.a.23635

40. Ackermann, J. et al. Metastasizing melanoma formation caused by expression of activated N-RasQ61K on an INK4a-deficient background. Cancer Res. 65, 4005-4011 (2005).

41. Damsky, W. et al. mTORC1 activation blocks brafV600E-induced growth arrest but is insufficient for melanoma formation. Cancer Cell 27, 41-56 (2015).

42. Dhomen, N. et al. Oncogenic Braf Induces Melanocyte Senescence and Melanoma in Mice. Cancer Cell 15, 294-303 (2009).

43. Krimpenfort, P., Quon, K. C., Mooi, W. J., Loonstra, A. \& Berns, A. Loss of p16Ink4a 
confers susceptibility to metastatic melanoma in mice. Nature 413, 83-86 (2001).

44. Abdel-Malek, Z. A. et al. Melanocortins and the melanocortin 1 receptor, moving translationally towards melanoma prevention. Arch. Biochem. Biophys. 563, 4-12 (2014).

45. García-Borrón, J. C., Abdel-Malek, Z. \& Jiménez-Cervantes, C. MC1R, the cAMP pathway, and the response to solar UV: extending the horizon beyond pigmentation. Pigment Cell Melanoma Res. 27, 699-720 (2014).

46. Cook, A. L. et al. Human Melanoblasts in Culture: Expression of BRN2 and Synergistic Regulation by Fibroblast Growth Factor-2, Stem Cell Factor, and Endothelin-3. J. Invest. Dermatol. 121, 1150-1159 (2003).

47. Cook, A. L., Boyle, G. M., Leonard, J. H., Parsons, P. G. \& Sturm, R. A. BRN2 in melanocytic cell development, differentiation, and transformation. in From Melanocytes to Melanoma: The Progression to Malignancy 149-167 (Humana Press, 2006).

48. Burian, E. A. \& Jemec, G. B. E. Eruptive Melanocytic Nevi: A Review. Am. J. Clin. Dermatol. 20, 669-682 (2019). 
Reporter +

CMV-Luciferase

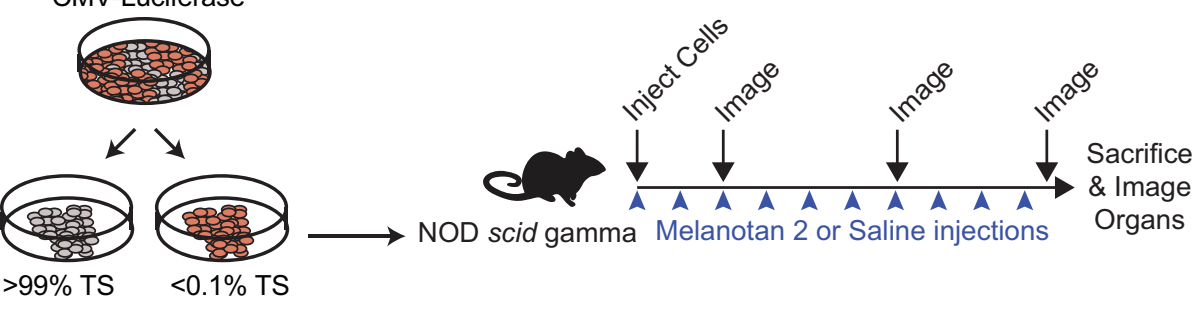

b

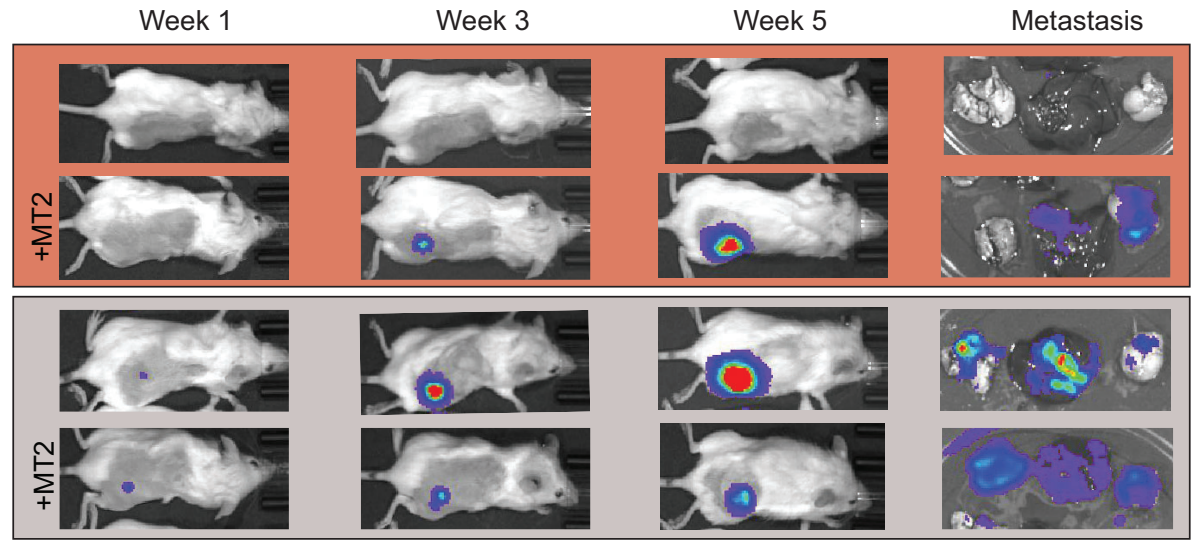

(2)
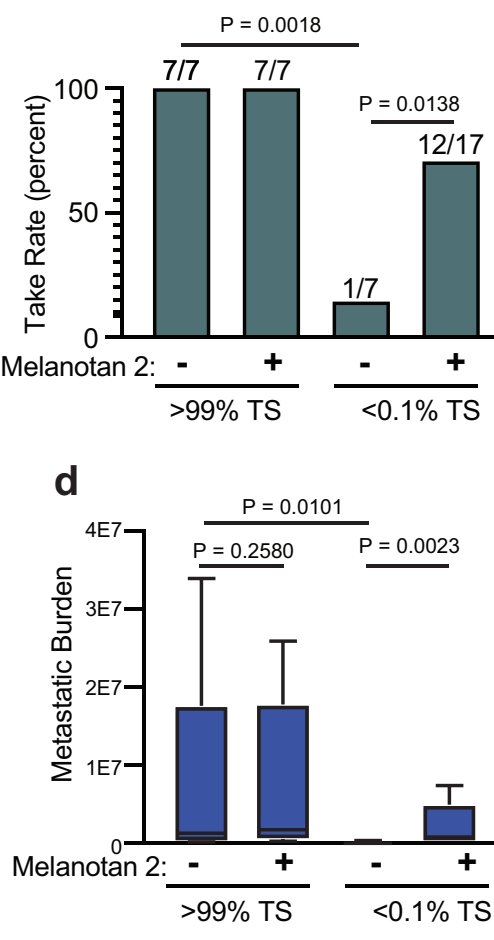

Figure 4 

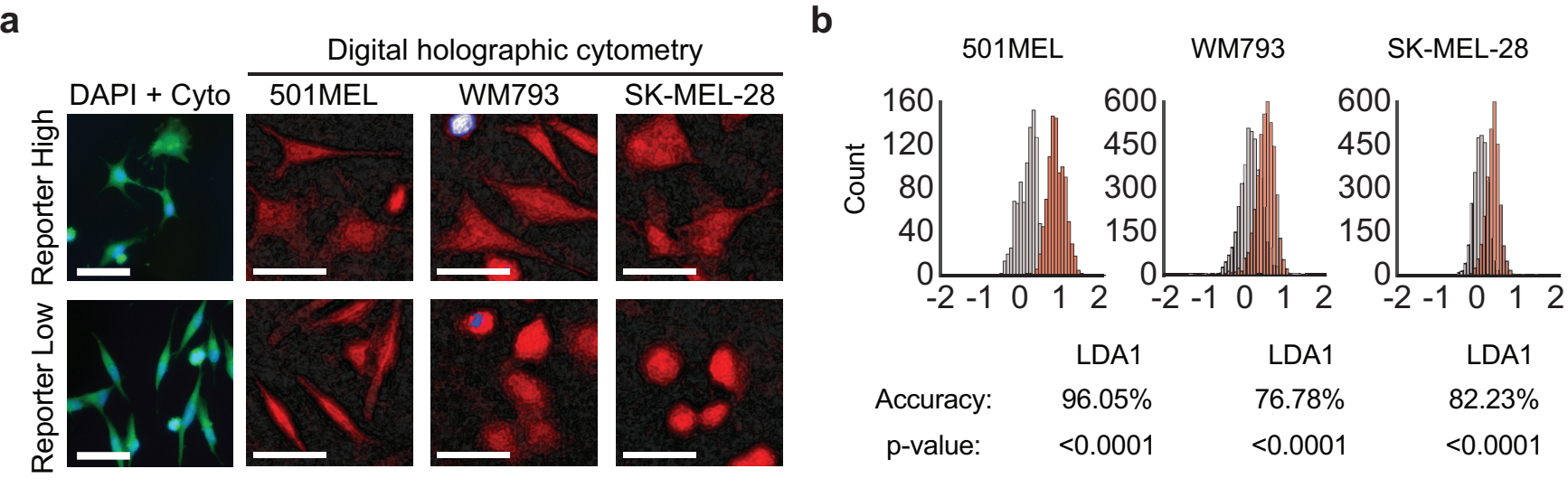

$\begin{array}{cccr} & \text { LDA1 } & \text { LDA1 } & \text { LDA1 } \\ \text { Accuracy: } & 96.05 \% & 76.78 \% & 82.23 \% \\ \text { p-value: } & <0.0001 & <0.0001 & <0.0001\end{array}$

$\underline{\text { WM793 }}$ 501MEL SK-MEL-28 $\mathrm{RH} \quad \mathrm{RL} \quad \mathrm{RH} \quad \mathrm{RL} \quad \mathrm{RH} \quad \mathrm{RL}$

POU3F2

HSP90

\section{d}

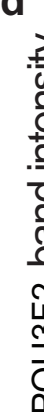

e

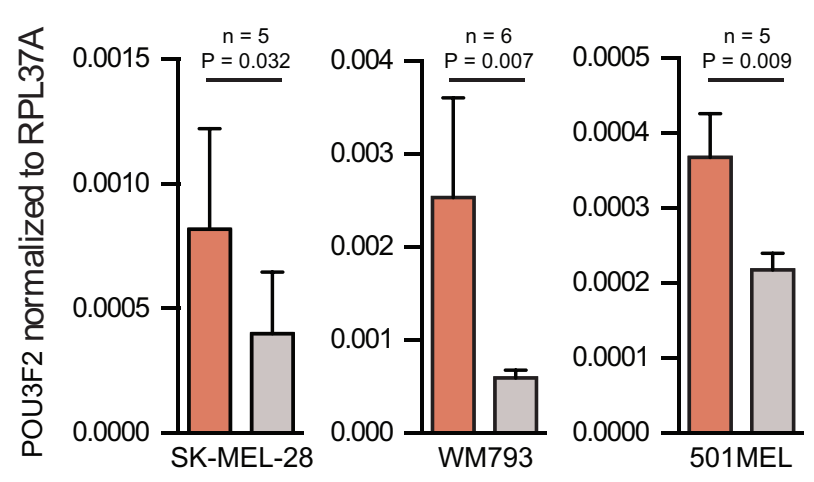

g

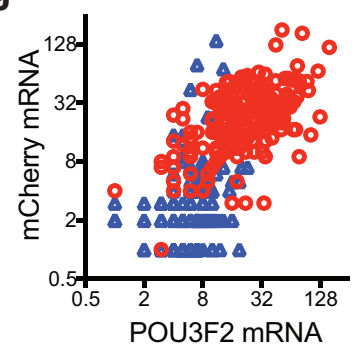

DAPI POU3F2 mCherry

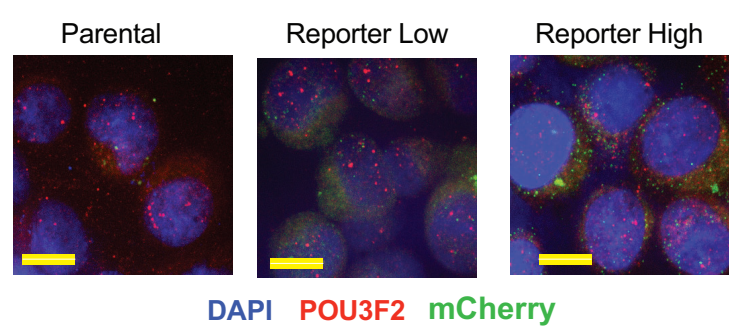

- Reporter High $\Delta$ Reporter Low

Figure S2 


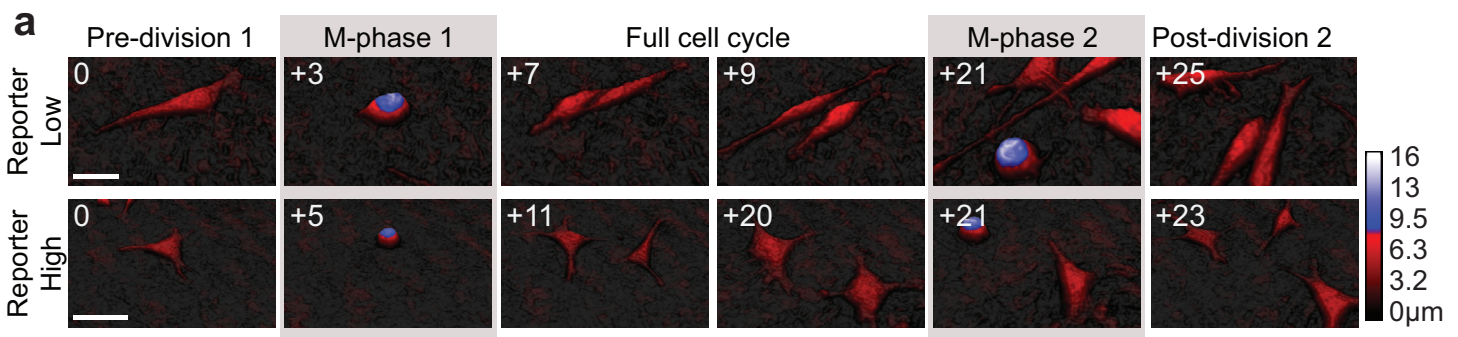

b
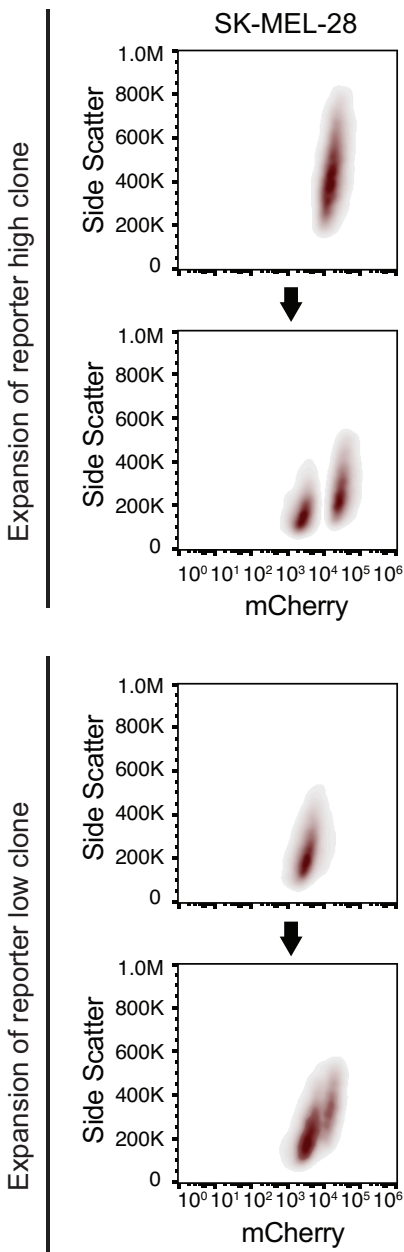

WM793
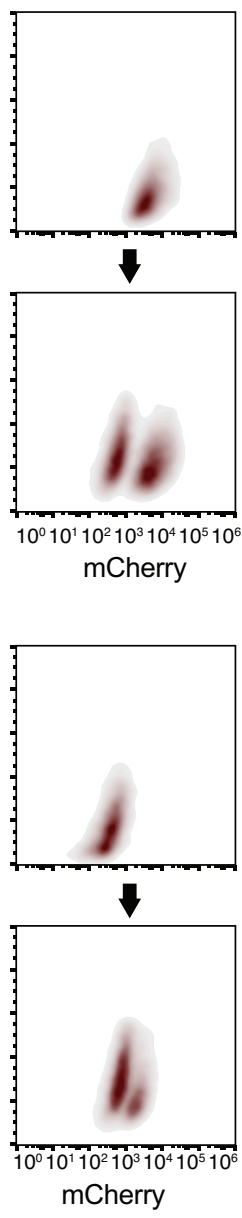

C

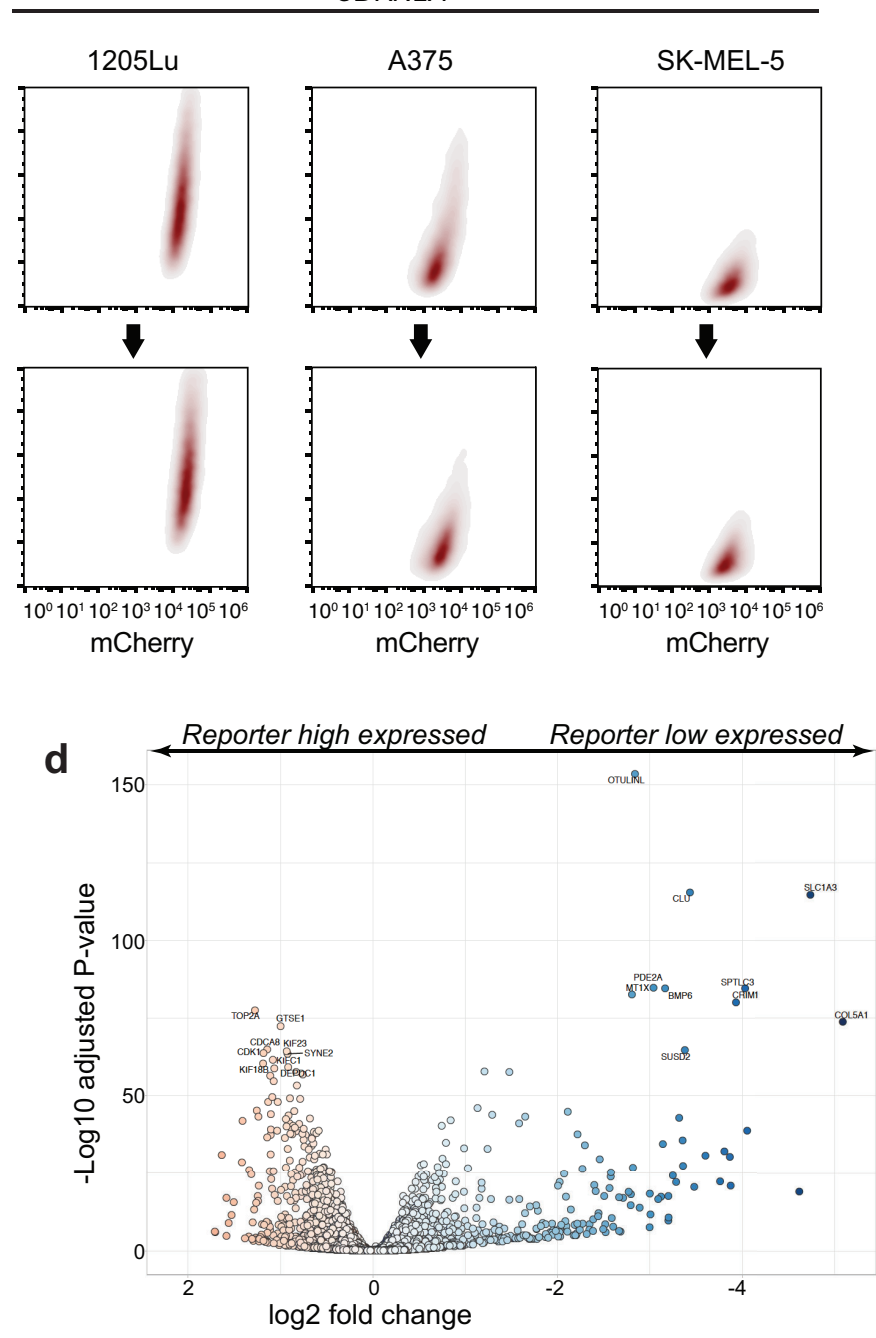


a

Change in TS population (delta/initial) after Melanotan 2 exposure

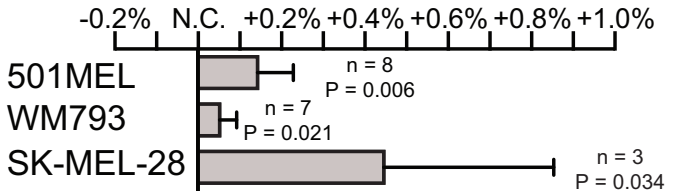

b

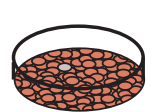

$<0.1 \%$

Reporter Low

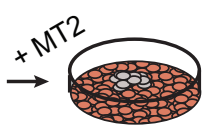

$<5 \%$

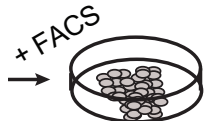

Isolated MT2-induced

Reporter Low

Reporter Low

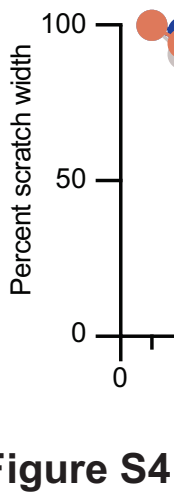


a. Development

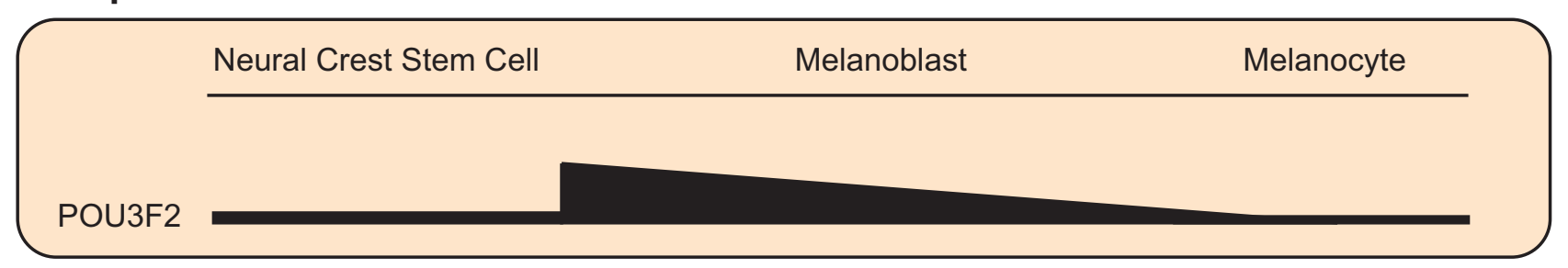

b. BRAF ${ }^{\mathrm{V} 600 \mathrm{E}}$ melanoma

Establishment of POU3F2-MITF axis

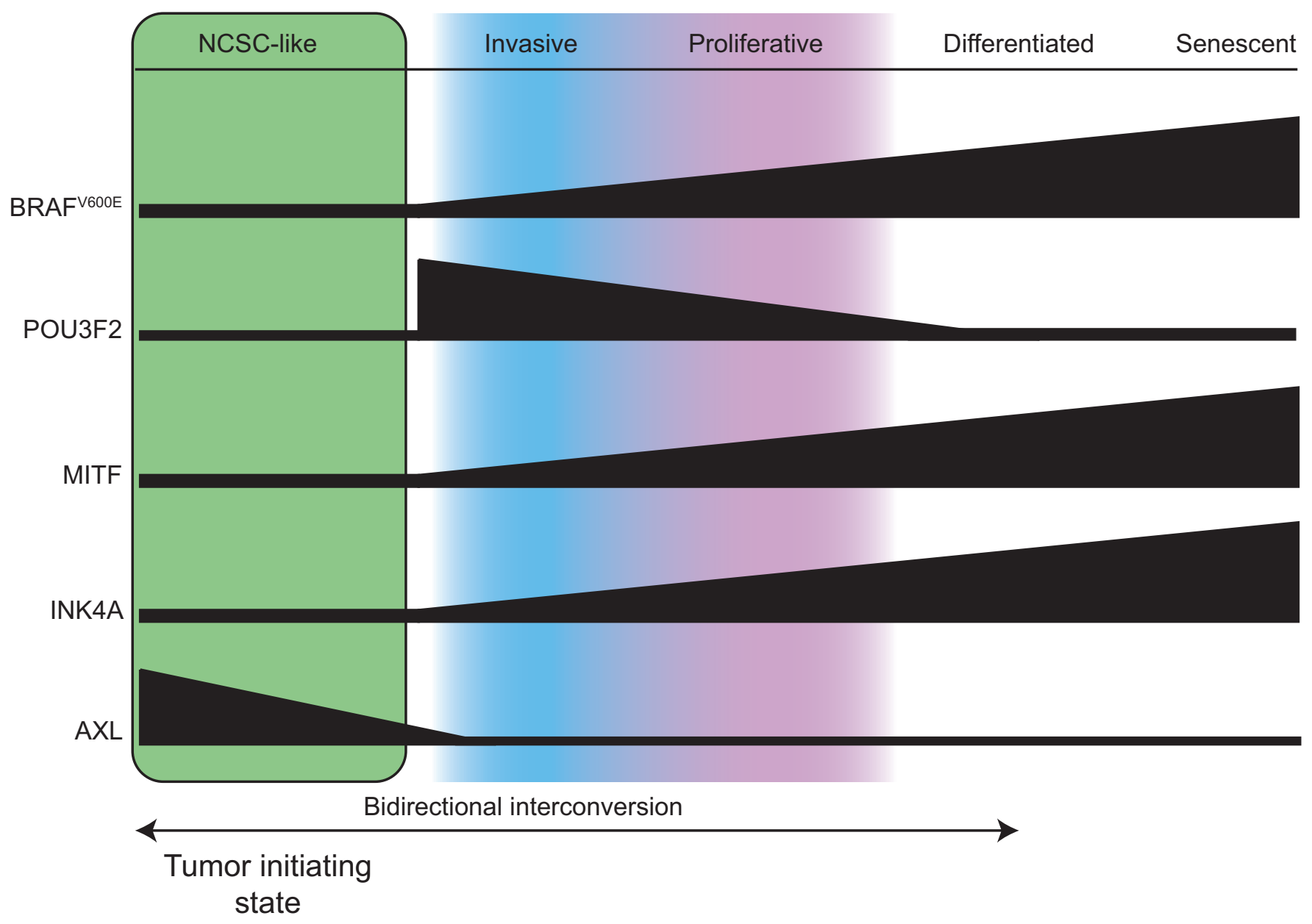

Figure S5 